\title{
Etnicidad, género y educación superior. Trayectorias de dos mujeres arhuacas en Colombia
}

\author{
Ethnicity, gender and higher education. Trajectories \\ of two Arhuaco women in Colombia
}

\author{
Angela Santamaria / angela.santamaria@urosario.edu.co \\ Universidad del Rosario, Colombia
}

\begin{abstract}
Two main questions are addressed in this text: how have these indigenous feminine leaders faced cultural restrictions to meet the demands of public life? How have they created a diverse amalgam of capital and knowledge as part of their leadership? The main theoretical elements used in this work are: the sociological perspective and field theory of Bourdieu, studies on intersectionality by Lorde (1984) and Pratt-Clarke (2010), the studies on higher education of Gil (2005), Choque (2015) and Mato (2015), and finally, the feminine leadership studies of Lumby and Coleman (2007). Our methodological approach is the reconstruction of two scholarly and professional personal trajectories from an intersectional perspective.
\end{abstract}

Key words: women, indigenous peoples, intercultural education, leadership, background.

Resumen: Nos preguntamos en este texto, ¿cómo han enfrentado estas lideresas las restricciones culturales frente a las exigencias de la vida pública? ¿Cómo han creado una amalgama de capitales y saberes diversos en el marco de sus liderazgos? Los principales elementos teóricos utilizados en este trabajo son: la perspectiva sociológica y la Teoría de los campos de Bourdieu, los estudios sobre interseccionalidad de Lorde (1984) y Pratt-Clarke (2010), los estudios de Gil (2005), Choque (2015) y Mato (2015) sobre educación superior intercultural, y finalmente los estudios sobre liderazgos femeninos de Lumby y Coleman (2007). Metodológicamente trabajamos a partir de la reconstrucción de dos trayectorias personales escolares y profesionales desde una perspectiva interseccional.

Palabras clave: mujeres, pueblos indígenas, educación intercultural, liderazgo, trayectorias. 


\section{Introducción}

Este artículo busca reconstruir las trayectorias sociológicas de dos mujeres indígenas del pueblo arhuaco egresadas de la educación superior colombiana. Estas historias son inéditas en los estudios colombianos sobre educación superior y pueblos indígenas en Colombia. Las experiencias de Luz Elena Izquierdo y Ati Quigua, madre e hija, al igual que muchas de las historias de las lideresas indígenas colombianas, han sido muy poco trabajadas. El análisis de estos materiales nos permite comprender las relaciones de dominación, rupturas y resquebrajamiento del poder masculino y líneas de acción al interior del pueblo arhuaco, y en los espacios políticos o institucionales por los que ellas han transitado.

Adoptamos una perspectiva de interseccionalidad en el marco de las luchas de las mujeres indígenas a nivel individual o colectivo para observar cómo su capital escolar y étnico les ha permitido abrirse un espacio político a nivel comunitario o institucional. Para ello utilizamos los trabajos de Lorde (1984), Gil (2005), Choque (2015) y Mato (2015). Trabajamos a través de la técnica de las trayectorias de vida, a partir de una mirada microsociológica, observando el peso de las fuerzas estructurales y también las líneas de ruptura inéditas de las actoras en el marco de los horizontes sociales posibles. Las preguntas que guían esta investigación son las siguientes: ¿Cómo enfrenta una lideresa indígena las restricciones culturales frente a las exigencias de la vida pública? ¿Cómo crea a través de una amalgama de capitales y saberes diversos liderazgos alternativos?

Partimos de un análisis de la relación entre educación superior, etnicidad, género y liderazgo desarrollados por Gil (2005). Como lo afirman Lumby y Coleman (2007), los estudios sobre educación superior y diversidad son muy amplios, pues esta categoría es muy vasta y hasta "camaleónica". Los estudios sobre la interfase entre liderazgo, diversidad y educación superior existen en varias regiones del mundo. Sin embargo, el cruce de estos estudios con la categoría de género es muy reducido. Este tipo de pertenencias aparecen como recursos o restricciones que es importante tener en cuenta para generar lecturas críticas de las prácticas de educación superior interculturales, que permitan generar políticas y líneas de acción, viables y pertinentes.

Igualmente, en el marco de estos estudios, como lo afirma Drucker (1997), se ha trabajado privilegiando la perspectiva del liderazgo de educadores y directivos universitarios, sus acciones y prácticas. En este artículo, retomando los trabajos de Mateos (2015), quisiéramos enfatizar en la perspectiva 
de los egresados. Adicionalmente, es importante resaltar que muchos de los estudios situados en esta interfase, se encuentran atravesados por la tensión entre dos polos antagónicos.

Así, se da el enfrentamiento entre perspectivas como las del feminismo radical, y los estudios críticos étnicos y de la raza, y los de la educación popular. Para este grupo de estudios, la meta-narrativa del patriarcado es la representación de la opresión de todas las mujeres y su rol en las estructuras primordiales (como la familia, la sociedad, la sexualidad y el trabajo). Estos roles sólo pueden cambiar mediante prácticas disruptivas de las trayectorias tradicionales de las mujeres y la incorporación de prácticas como la planificación, el acceso a la educación superior y la opción del divorcio.

Autores como Lorde (1984) y Pratt-Clarke (2010) hablan de la necesidad de comprender las consecuencias estructurales y las dinámicas de funcionamiento cuando confluyen dos o más prácticas de dominación: como las sexistas y racistas. Esto desemboca en una tensión entre experiencias de inclusión/exclusión educacional. Esta reflexión va de la mano de la necesidad de fortalecer las políticas y acciones afirmativas, a través de perspectivas educativas con enfoque de interseccionalidad. Dicha visión ha sido poco trabajada por visiones sobre la exclusión de grupos vulnerables de la educación superior como la feminista, indigenista o los estudios sobre la raza.

Hay que resaltar aquí el importante trabajo histórico desarrollado por Bermúdez (2015) sobre la educación de las mujeres en los países andinos. Algunas autoras colombianas como Ulloa et al. (2005) y González (2013) han analizado sobre todo los procesos de resistencia y de construcción de memoria de las mujeres indígenas. Principalmente González trabaja a partir de las biografías tan usadas por autores como Ken (2008), Rabouin (2000), Wing y Willis (1999) en otros contextos. Otras autoras como Gil (2005) han trabajado sobre las historias de vida de estudiantes indígenas en la ciudad de Bogotá, originarias de los pueblos Wayúu y Kamentsa. Sin embargo, el campo de estudios está apenas en formación.

Siguiendo a Wade (1997), partiremos de la categoría “indígena” desde una historia local específica: la de Ati Quigua y su madre Luz Elena Izquierdo. Es interesante resaltar que la visión oficial del "indígena” aparece como desencarnada y asexuada, reconociendo derechos especiales a grupos muy heterogéneos cultural, sexual y económicamente. Otros autores latinoamericanos como Arturo Escobar, Sonia Álvarez y Evelina Dagnino (2001) han mostrado en sus trabajos cómo durante las últimas décadas los conflictos que enfrentan los nuevos movimientos sociales los han llevado a imaginar y pro- 
poner prácticas en búsqueda de democracias más radicales, que trasciendan el nivel formal institucional, a través de acciones colectivas que interpelen los sistemas políticos y constitucionales.

La historia de estas mujeres muestra un vínculo constitutivo entre cultura y política. Los trabajos de Bourdieu (1989) ofrecen herramientas teóricas y metodológicas muy pertinentes para analizar el caso de este grupo de mujeres indígenas. La noción de "campo" como un espacio relativamente autónomo, como microcosmos dotado de sus propias lógicas de funcionamiento, es para nosotros muy fecunda. La noción de campo simbólico (educativo, político, del conocimiento, del Estado, étnico) es fundamental para nuestro análisis, pues nos permite situarnos en el cruce de diferentes disciplinas y objetos de estudio.

El concepto de campo(s) y las interacciones entre sus diversas expresiones permite que "la causa indígena" no sea reducida a lo meramente cultural, o a lo jurídico o político. Las herramientas teóricas del campo se vuelven concretas a partir de la utilización de la metodología de las trayectorias de vida. El autor fue muy crítico con la noción de historia de vida y biografía, y su tendencia a la apología de los protagonistas.

Desde este punto de vista enfatizamos en la necesidad de sociologizarlas mediante las trayectorias. Igualmente, ocupamos la observación etnográfica de larga duración para la construcción de éstas. Autores como Bosa y Wittersheim (2009) se han inspirado en dichas propuesta para analizar problemáticas indígenas relacionadas con la educación y la entrada al campo político en escenarios poscoloniales.

En este sentido, la reconstrucción de las trayectorias de estas dos mujeres arhuacas, desde nuestro punto de vista atípicas, busca articular lo macro y lo micro del proceso de egreso y entrada al campo político. Hemos trabajado con ellas compartiendo su vida cotidiana y observando sus procesos políti$\cos ^{1}$ durante aproximadamente cinco años. El presente trabajo en profundidad se ha materializado gracias a conversaciones y a un grupo de entrevistas etnográficas formales. Nos interesa resaltar no sólo lo micro y lo macro, sino destacar las tensiones, las relaciones de poder, pero también las posibilidades y éxitos que el acceso a la educación superior les ha posibilitado.

1 Desde el año 2009, hemos co-organizado con ellas cuatro diplomados sobre Educación y liderazgo intercultural en la Sierra Nevada de Santa Marta. Ellas han participado como formadoras en los procesos. Este escenario ha sido una plataforma para la observación y la recolección de datos. 
Aquí son muy importantes las ideas de La Red de Modernidad/Colonialidad, para revisitar la cuestión del poder en la modernidad mediante la ubicación de sus orígenes en la conquista de América, poniendo un especial énfasis en la estructuración del poder a través del colonialismo y las dinámicas constitutivas del sistema-mundo moderno/capitalista. Nos interesa resaltar especialmente el trabajo de Quijano (2000) sobre la necesidad de subvertir la categoría "indígena” como forma de control colonial. Se vuelve aún más una necesidad, puesto que a pesar de la subversión del estigma movilizado por la categoría mediante el reconocimiento oficial de los derechos indígenas y el proceso de constitucionalización del derecho a la diversidad étnica y cultural, y de la educación indígena, en la década de 1990, en Colombia las mujeres han sido las grandes ausentes en la historia política.

La memoria reconstruida desde las organizaciones y los pueblos ha dejado a sus mujeres invisibilizadas, como una mención, o una nota al pie de página. Retomando a Korol (2007), pensamos que la "colonialidad del poder" es reproducida indirectamente, por autores como Le Bot (2004), Gros (2000) y Laurent (2005), quienes la refuerzan al centrarse en la historia de los líderes hombres, como una sucesión escalonada de acciones de grandes dirigentes.

Existen trabajos muy interesantes como el de Gil (2005) sobre 14 estudiantes y egresadas indígenas de instituciones de educación superior en la ciudad de Bogotá. Sin embargo, el énfasis de la autora no se centra en los liderazgos femeninos indígenas. Por ello, proponemos una reconstrucción de las trayectorias sociológicas de estas dos lideresas arhuacas, las cuales se han articulado con observaciones etnográficas en la Sierra Nevada de Santa Marta y en escenarios nacionales.

\section{Luz Elena Izquierdo. Primera mujer arhuaca en incursionar en el alto gobierno}

\section{Educación Superior y Misiones}

Luz Elena Izquierdo Torres nació en feurwa en la Sierra Nevada de Santa Marta. Fue la primera universitaria del pueblo arhuaco. Su familia por línea materna mantuvo la tradición arhuaca gracias a que permanecieron lejos de la Misión Capuchina en las nieves de la Sierra. Según los estudios de Bosa (2012), los monjes capuchinos se instalaron en Nabusímake y construyeron un orfanato. Desde 1914 hasta principios de la década de 1980 aplicaron estrategias de evangelización mediante el rapto de niños y niñas arhuacos y el internamiento forzado en un primer momento. 
Este tema ha sido trabajado por Gil (2005) en otras zonas del país como la Guajira y el Putumayo. Como lo afirma Bosa (2015) para el caso del pueblo arhuaco, posteriormente el internamiento se hacía de manera voluntaria por parte de las familias "hijas" de dicha institución. Autores como Bosa (2012) han hecho estudios comparativos sobre la historia de la "generación robada" en Australia y en Colombia.

El caso de Luz Elena ilustra perfectamente estas lógicas misioneras globales a nivel local. Sus abuelas tuvieron destinos sociales y trayectorias muy diversas. Simeona y Cornelia se criaron desde su cultura. En los archivos del Colegio de Nabusímake elaborados por Bosa (2012), estas mujeres arhuacas eran, a su vez, muy distintas entre sí. Cornelia escapó de la Misión y se crió en las Nieves lejos de la influencia de los curas. Simeona, además de cuidar a sus hijos y su finca, ejerció actividades económicas como la venta de sus animales, hizo denuncias ante las autoridades policiales y el Consejo de Ancianos, es decir, se desempeñó como una lideresa contemporánea. Eloisa, por el contrario, fue raptada y educada en la Misión Capuchina, al igual que Blacina. Estos acontecimientos históricos generaron una división interna en la familia.

Mita, la madre de Luz Elena, hija de un matrimonio conformado en la Misión, pasó por dicha institución de manera voluntaria, pues su madre Blacina había sido educada por los curas. Las Izquierdo son de feurwa, donde están los cerros para hacer pagamento al agua. ${ }^{2}$ Sin embargo, las Izquierdo son muy diversas entre sí y han tenido acceso diferenciado a la educación misional. En palabras de Luz Elena refiriéndose a su tía Cornelia:

A ella y su familia les daba miedo venir a vernos en la zona donde estábamos, no hablaban español, y decían que "ya éramos civilizados". Por otro lado, mi abuela la que sí fue criada en la Misión, contaba la crueldad de los curas. Narraba cuando le quitaron la manta, le prohibieron hablar la lengua Ika. Aunque allá aprendió a leer y a escribir, lo que más le enseñaron fue religión. Ya en edad adulta, le consiguieron pareja, y los casaron por la Iglesia católica. Salían casados, con una nueva familia a defender esa cultura de los curas. Pero es complicado, a ella la casaron con mi papá que es Mamo, pero él no pasó por la Misión. Él y ella estaban aferrados a los Mamos, su abuelo y bisabuelo habían sido Mamos. Ella siempre ha sido una mujer arhuaca preocupada por sus tradiciones (Entrevista a Luz Elena Izquierdo, Bogotá, 2013).

2 El pueblo arhuaco funda su cosmovisión sobre cuatro elementales (agua, fuego, aire y tierra). Todo miembro de la comunidad debe rendirle tributo a estos elementales. Una de las formas de hacerlo es a través de ceremonias de limpieza energética en los cerros y montañas que los representan. 
Según la tradición arhuaca, a las mujeres no se les permitía estudiar. Sin embargo, el tema fue casi una obsesión desde pequeña para Luz Elena. Los libros y el estudio fueron un refugio para enfrentar muchos de sus conflictos durante la niñez. Su padre no fomentó esta vocación, pero -lo más importante- tampoco la prohibió. No obstante, Luz Elena tuvo que enfrentar otro dilema cultural, pues fue escogida desde el vientre por una de sus abuelas para quedarse con ella, aprender la lengua y la cultura.

\section{La escuela formal vs la cultura}

Durante su estadía con la abuela, Luz Elena niña pastoreaba ovejas en los cerros. A pesar de que disfrutaba de su abuela y sus enseñanzas, quería ir a la escuela. Un día, cuando su padre fue a visitarla escapó, y lo esperó adelante en el camino para que la llevara con su mamá. Hizo su primaria en feurwa. Unas seglares españolas fueron sus profesoras. Éstas propusieron a los padres de Luz Elena, cuando ella tenía diez años, llevarla a España a estudiar. A pesar del entusiasmo de la madre y la hija, su padre dijo rotundamente que no. Para cursar el bachillerato, fue enviada junto con un grupo de niñas a estudiar a Manaure. El hito tal vez fue la llegada del padre Javier Rodríguez. Este acontecimiento individual obedece a cambios estructurales que se estaban gestando a nivel nacional e internacional. Como lo hemos trabajado en otras investigaciones (Santamaria, 2008), la figura del padre en cuestión ilustra procesos de reconocimiento de derechos indígenas a nivel internacional, en el marco de la Declaración de Barbados y la OIT durante las décadas comprendidas entre 1950 y 1970. En palabras de Luz Elena:

Me acuerdo que llegó ese cura muy joven, y muy rápidamente se ubicó en el tema, identificó los problemas y la cultura. Para él, no era normal, ni estaba bien lo que estaban haciendo los capuchinos. La llegada del padre Javier coincidió con que yo iba a hacer el bachillerato y él nos consiguió un cupo en un internado cerca de Valledupar. Para mantenernos, el padre cogió y vendió el ganado de la Misión porque veía cómo se seguía acumulando éste, y los indígenas trabajando para los misioneros, y ninguno avanzaba en los estudios. Así, sacó el primer grupo de arhuacos a estudiar a colegios en Manaure. Todos éramos hijos de los indígenas que trabajaban para la Misión o habían estado en ella (Entrevista Luz Elena Izquierdo, Bogotá, 2013).

Así fue como, paradójicamente, esta mujer se convierte en una de las primeras bachilleres arhuacas ${ }^{3}$ con el apoyo del padre Javier, quien llegó como

3 Para lograr su manutención, vivió y se hizo cargo del cuidado de niños, en la casa de su familia vallenata de la élite política: los Villazón. De esta manera logró terminar sus estudios secundarios. 
director de la Misión. Sin embargo, las dificultades y enormes sacrificios para terminar sus estudios no la eximieron nunca de sus compromisos y obligaciones familiares y culturales. El liderazgo de Luz Elena nació al lado del fogón, escuchando las historias de su padre, ayudando en la cocina familiar y trabajando en los eventos comunitarios en donde se reúnen más de 300 personas. Pero este liderazgo no acaba allí. También se teje en las aulas de clase, en las visitas a las comunidades más retiradas para dar atención en salud, en el trabajo de consulta y en los trabajos espirituales con los Mamos. El liderazgo femenino está asociado con el trabajo comunitario, ser hablante de su lengua nativa, o tener equivalentes culturales, la maternidad, el cuidado del marido y de la familia. Igualmente, el tejido de las mochilas para el marido y los hijos. Es allí, a través de las mochilas, como el hombre muestra estatus, poder.

El militantismo indígena está atravesado por una división social y sexual del trabajo político, que genera en su interior una jerarquía de objetos y causas sociales. La división de los roles de género y su relación con la educación ha sido estudiada por Gil (2005). Sin embargo, el pueblo arhuaco posee características específicas en relación con el liderazgo, pues los líderes y lideresas acuden con sus hijos y compañeras a las reuniones. Sólo aquellos que están en pareja, que tienen hijos, tienen por lo general legitimidad ante la comunidad para hablar, para liderar.

En palabras de Geremías Torres, el representante a nivel nacional del Pueblo arhuaco, "un hombre, una mujer que no tenga familia, que no se haya hecho responsable de hijos, finca (...) no puede ser un buen líder" (Diario de campo, Nabusímake, diciembre, 2013). Por todo lo anterior, analizamos en el siguiente apartado algunas dimensiones de la trayectoria emocional de Luz Elena, para mostrar cómo durante toda su vida ha tenido que ejercer múltiples roles y "dobles jornadas" para ser la lideresa que hoy es.

\section{El ingreso a la Universidad Javeriana de Bogotá}

Luz Elena se radicó en Bogotá a finales de la década de 1970 en pleno despertar del movimiento indígena, como lo afirman Le Bot (2004) y Gros (2000). En esa época no había convenios entre organizaciones indígenas y universidades, ni programas de becas, como existen actualmente en Colombia y otros países. Los procesos actuales han sido estudiados en profundidad por Gil (2005). Para Luz Elena, la perspectiva de egresar era mínima, y más para una mujer indígena con hijos. Sin embargo, ella lo logró. Luz Elena se casa con un Guanano del Vaupés, Quigua, originario de una familia indígena explotada por los caucheros. En esas circunstancias los procesos de socialización y de 
escolarización de la pareja tuvieron lugar en regiones distintas, pero bajo la "colonización de las mentes" por parte de los misioneros.

Luz Elena comenzó sus estudios universitarios en los años ochenta. La discriminación en las grandes ciudades era muy fuerte, y sobre todo en un contexto como el universitario. Los misioneros generaron en sus procesos de educación sistemática la formación de "poderes coloniales", aún vigentes en Colombia, para retomar los términos de la afroamericana Pratt-Clarke (2010). Existe continuidad en la permanencia de dichos patrones, como lo muestra Gil (2005) para el caso de las estudiantes Wayúu y Kamentsa en la ciudad de Bogotá.

Para hacerse cargo de la crianza de su primera hija, Luz Elena regresa a la Sierra. Se instala en feurwa, donde vive la abuela Mita. Luego regresa a seguir sus estudios y tiene un segundo hijo, que se cría también con la abuela. Mita nunca perdió la lengua, pero no se la enseñó a sus hijos, porque en la Misión era prohibido. También perdió su vestido tradicional a pesar de venir de un linaje de Zagas ${ }^{4}$ de faja negra. Para terminar sus estudios, Luz Elena tuvo que separase de sus hijos y salir de su territorio por varios años.

La vida en la capital de Colombia sin becas ni apoyo familiar no era una posibilidad para estar con ellos. Después de concluir sus estudios, Luz Elena se trasladó al Vaupés durante aproximadamente diez años con Quigua. Tras enfrentar los celos, la violencia física y psicológica de su esposo, decide irse a la Sierra con sus hijos. Durante su estadía en la Amazonia trabajó en un hospital. A nivel nacional e internacional se estaban gestando las discusiones preliminares del Convenio 169 de la OIT, el cual sería aprobado en 1989.

En trabajos anteriores hemos analizado cómo a nivel nacional, durante este periodo, se consolidaron los primeros esbozos de la etnoeducación y de la salud "propia" (Santamaria, 2007). Los hijos de Luz Elena: una niña, Ati, y un niño, Norey, crecen y se escolarizan entre la Sierra Nevada y la Amazonia colombiana. Aquí se da una de las rupturas biográficas y culturales más fuertes en la trayectoria de Luz Elena: el divorcio y el asumir a sus hijos en un modelo monoparental. Ante los peores pronósticos de la abuela, "que nadie la iba a querer, que había que aguantar" perspectivas muy comunes en mujeres indígenas de su edad, la lideresa reconstruye su vida laboral y emocional. Luz Elena subvierte el dispositivo de control del matrimonio y la preservación de la familia, utilizado en un principio por los misioneros y luego por las

4 El pueblo Wiwa, también de la Sierra Nevada de Santa Marta, tiene autoridades espirituales mujeres que juegan un rol como el del mamo en el pueblo arhuaco. 
autoridades indígenas para el control de las mujeres y hombres, como espacio privilegiado para la reproducción de la cultura.

Su trayectoria nos permite adentrarnos en los tejidos de los patrones de la "colonialidad del poder" a través de una experiencia concreta. Mediante el acceso a la educación y al trabajo, esta mujer hace rupturas biográficas inéditas en su familia y en la sociedad. Así, prosigue su formación universitaria como enfermera en la Universidad Javeriana y luego su Maestría en Salud Pública, lo cual le permite contribuir a su comunidad como miembro del Comité de Salud y, posteriormente, como fundadora y primera gerente de la empresa de Salud Indígena Dusakawi. Igualmente, rehace su vida con un indígena del Cauca colombiano, con quien tendrá años después una última hija: Dunen Muelas. Por amenazas de grupos paramilitares, tiene que renunciar y desplazarse a Bogotá.

El análisis de Quijano arroja luces para comprender la "subalternización” de la subjetividad de Luz Elena (Quijano, 2000). Sin embargo, nos interesa resaltar, como lo mostraremos, las posibilidades de autonomía y las resistencias que emergen en este entorno, desde su experiencia personal.

\section{La incursión en el alto gobierno}

Esta lideresa fue la primera mujer indígena en incursionar en el gobierno nacional. Su capital étnico y escolar fueron fundamentales en el momento de su nombramiento. Era una de las mujeres indígenas más formadas del país. Además, muy cercana a los dos primeros ex constituyentes indígenas de Colombia de 1991. Era una época de difícil diálogo entre los pueblos indígenas y el gobierno nacional. Bush et al. (2005) han denominado a este proceso "racismo institucional". Sin embargo, la entrada de la lideresa al gobierno se da en el contexto de aplicación de la Constitución de 1991. Ésta reconoce derechos especiales a los pueblos indígenas y se convierte en una de las cartas constitucionales más progresistas en América Latina. Sin embargo, si bien a nivel macro se estaban dando estos cambios, en la vida cotidiana la realidad era otra. En términos de la lideresa:

Me acuerdo cuando la de Recursos Humanos me llevó a mi oficina y dijo: "Aquí está, ésta es la nueva directora", y ya. Y cuando yo veo tenía como a veinte personas a mi cargo. En la gerencia de la empresa prestadora de salud Dusakawi yo manejaba 120 personas, yo estoy formada para organizar equipos humanos, esa parte no me parecía complicada. Sin embargo, la Dirección de Etnias era distinta. Había una discriminación contra la entidad, era la cenicienta, un quemadero, pues había mucha exigencia 
por parte del movimiento indígena. Hoy el nivel de la entidad es distinto, muchas veces estuve por renunciar (Luz Elena Izquierdo, Bogotá, 2013).

Y así fue, después de un año y medio renunció. Hoy se desempeña como asesora del director de etnias a nivel nacional. Desde su experiencia como mujer indígena, afirma que los distintos actores nacionales e internacionales han reclamado en los últimos años la presencia de las mujeres indígenas en los escenarios políticos; sin embargo, la situación no ha cambiado mucho, y sólo hay con ella otras dos mujeres en cargos de visibilidad.

\section{Ati Quigua. La primera Concejal indígena de Colombia}

\section{La escuela en feurwa y el Internado de Nabusimake}

La escuela de feurwa fue la primera fundada por profesores indígenas graduados de la Misión, el tío materno de Ati fue uno de los primeros maestros. Sin embargo, cuando ella ingresa a la Escuela, ya la Misión había sido expulsada de Nabusímake, y el pueblo arhuaco asumía la educación indígena. Como lo afirma Choque (2015), a nivel nacional en la región andina se estaba trabajando en la implementación de políticas de etnoeducación o de educación indígena, y a nivel internacional se gestaba la consolidación del grupo de trabajo para pueblos indígenas de las Naciones Unidas.

Esto implicó para el internado arhuaco implementar ajustes curriculares, la autonomía de los maestros indígenas y la salida de las maestras españolas. Ati vivió entonces un proceso de escolarización muy distinto al de su madre. Éste se desarrolló en el territorio arhuaco y en el Vaupés, profundizando en la historia del proceso político arhuaco las luchas históricas de los dirigentes, y durante un periodo pre Constitución de 1991, en el que "ser indígena" comenzaba a conocer una subversión del estigma. Para su formación en secundaria, ingresó como interna, durante seis años, al Colegio Agropecaurio Arhuaco, en las antiguas instalaciones de la Misión Capuchina.

Éramos 45 internas y unos 60 internos. Se estaban demoliendo las construcciones de la Misión. Pasamos de una arquitectura española a un edificio suizo, donado por la cooperación, y aportes en trabajo y material de las familias de los internos. A las cinco de la mañana sonaba una campana que quedó de la época, para irnos a bañar al río. Teníamos profesor de afuera y de la comunidad, por ejemplo el de lengua materna. A la

5 La Constitución de 1991 fue una de las primeras en la región, después de la brasilera que reconoció a los pueblos indígenas como sujetos especiales de derechos colectivos. Igualmente, reconoció el Estado colombiano como multicultural y pluriétnico. 
una terminábamos la jornada escolar, y nos íbamos a la huerta y a cuidar los animales, conejos, cerdos, ovejas, vacas. Aprendíamos a desparasitarlos, a inyectarlos, a castrar, a soguear, a ordeñar. También sembrábamos hortalizas y papa (Entrevista Ati Quigua, Bogotá, 2012).

La educación en la Sierra estaba dirigida por el Mamu Kunchana Bingumu. Ati Seygundiba significa "Madre de los Buenos pensamientos". La disciplina del Colegio arhuaco reprodujo mucho de la cultura misionera. Aún hoy en el Manual de Convivencia "está prohibido enamorar", so pena de expulsión. Esta situación nos recuerda las palabras de Foucault, cuando afirma que "si la sexualidad está reprimida, es decir, destinada a la prohibición, a la inexistencia y al mutismo, el sólo hecho de hablar de ella y de su represión, posee como un aire de transgresión deliberada" (Foucault, 2002). Una de las estudiantes arhuacas de la generación de Ati que ya estaba terminando su secundaria para ser tal vez la primera arhuaca médica, fue expulsada por una carta de amor. Las costumbres frente a las relaciones de pareja están permeadas por la fuerte moral religiosa heredada, y su hibridación con concepciones culturales sobre las mujeres.

Para la comunidad arhuaca, el cuerpo de la mujer es de la comunidad, no de la mujer. Por ello, el Mamo genera dispositivos de saber-poder, de control sobre la sexualidad. Retomando las palabras de Longo (2007), éste establece formas de disciplinamiento sobre la hexis corporal (cómo sentarse, hablar, mirar). El tejido de la mochila, por ejemplo, es una práctica de concentración, silenciosa. En palabras de Ati:

Siempre está la mujer mirando hacia abajo. Una mujer que mira a los ojos es interpretada como alguien que está estableciendo un intercambio íntimo. Las mujeres llevan la mochila en la cabeza y están concentradas en ella, ninguna mujer se para frente a un hombre, eso se considera un mal comportamiento, una insinuación, todas se paran de medio lado, se orillan en el camino (Ati Quigua, Bogotá, 2013).

Los espacios de responsabilidad les exigen a hombres y mujeres estar casados. Existe la creencia de que la responsabilidad de gestionar la casa, y la familia, garantiza un buen liderazgo. Sin embargo, la organización y la comunidad no ofrece garantías para la participación de la mujer en los espacios educativos o de decisión. Como Chisholm (2001) lo ha observado en otras regiones del mundo, el matrimonio no es una garantía de participación. Tras varios años de seguir la trayectoria personal y política de Ati, hemos podido observar el nacimiento y consolidación de un liderazgo femenino desde el “cuidado", en palabras de Gilligan (1982) y Noddings (1988). 


\section{La politica y el matrimonio: tensiones entre dominación y oportunidades}

A partir de la experiencia de Ati, es claro que la educación superior es una oportunidad para corto-circuitar la dinámica y el rol de la mujer en la sociedad arhuaca y generar liderazgos en la diversidad. Es importante resaltar que en algunos casos el capital escolar y político acumulado, como en el de la campaña de Ati para el Senado de la República, y un trabajo colaborativo con hombres indígenas de su familia, como su hermano Norey Quigua, y los Mayores Jeremías Torres y Álvaro Torres, han generado posibilidades para que su proceso tenga el aval de la organización arhuaca. Esta experiencia de subversión de la división tradicional del trabajo político entre los sexos, desde una "acción afirmativa desde lo cotidiano", es importante para pensar ¿cómo podrían generarse casos similares, para otras mujeres indígenas? Y ¿cómo pasar de lo individual a acciones más colectivas?

$\mathrm{Al}$ respecto, Norey, hermano de Ati y quien forma parte de la organización arhuaca, afirma que tanto su madre y su hermana han tenido en momentos cruciales "detrás un gran hombre". Lo anterior denota que sin el "padrinazgo de un líder” es difícil que emerjan liderazgos de mujeres, pues son ellos quienes siguen conservando el poder.

Esta oportunidad, además, no está al alcance de todas las mujeres arhuacas y/o pertenecientes a otras etnias. El acceso a la educación y el apoyo de líderes indígenas está en muchos casos reservado a mujeres hijas de familia de líderes. En el caso de Ati, su primer matrimonio con Moisés Villafañe le permitió incursionar en el ámbito de la política electoral. Aquí es importante resaltar el trabajo de Laurent (2005), pues la participación política de Ati se da en el contexto del reconocimiento de las circunscripciones electorales indígenas. Su liderazgo se construye principalmente hacia afuera, lo que paradójicamente beneficia el proceso de interculturalidad, pero no genera transformaciones en el acceso a la educación y el liderazgo de las mujeres a nivel comunitario.

La experiencia de la lideresa muestra temáticas emergentes que deben ser analizadas. El tema, por ejemplo, del matrimonio y el egreso de la educación superior y el ingreso al campo laboral. Para el caso de Ati, el matrimonio fue una oportunidad de iniciar un proceso político; empero, los costos emocionales fueron muy altos, debido al perfil muy tradicional de su esposo. Puesto que el liderazgo indígena requiere ser reconocido por otros líderes hombres, en su caso se observa un efecto de reproducción de los líderes y de los liderazgos por linajes y sexo. 
Es así como a pesar de haber participado en política antes de entrar a la universidad y tener más experiencia que muchos hombres, no es elegida. $\mathrm{Al}$ respecto, su hermano Norey afirma: "Yo no creo en esos temas de desigualdad entre los géneros. Por ejemplo, Aida Quilcué, una gran autoridad indígena. Cuando la veo, yo no veo una mujer, veo una autoridad". Es decir, en el mejor de los casos se admite que una mujer valiente, audaz, se convierta en una "autoridad", la cual, sin embargo, debe esconder a la mujer. Lo anterior ocurre en el caso de Ati, quien posee un perfil político que busca el reconocimiento de las mujeres indígenas y campesinas. Después de su divorcio con su primer esposo, Ati rehace su vida con un político indígena (Gerardo Jumí), con quien tiene su primera hija: Gunnavia.

\section{El ingreso a la Universidad}

Ati pertenece a una segunda generación de mujeres para quienes ir a la Universidad era necesario y evidente. Cuando inicia sus estudios, existían en el país distintas oportunidades y programas de educación superior para pueblos indígenas. ${ }^{6}$ Inicia el pregrado en Administración Pública, en la Escuela

6 En el caso colombiano, hay varios procesos desde las organizaciones indígenas y a través de alianzas con Universidades “convencionales” (Mato, 2011). Por ejemplo, la Universidad Autónoma, Indígena e Intercultural (UAIIN) como consecuencia de más de treinta años de educación indígena del Consejo Regional Indígena del Cauca (CRIC). Esta institución, creada en 2003, ofrece licenciaturas (Administración y Gestión y Desarrollo propio, y Pedagogía Comunitaria, Derecho Propio) y Diplomados (en Proyecto educativo comunitario, en Currículo propio, Gestión Etnoeducativa, Familia y Equidad de Género y Políticas de la Salud). De estos programas, según Graciela Bolaños, Libia Tattay y Avelina Pancho, sobre 859 estudiantes egresaron 349 mujeres en 2007, frente a 510 hombres (citado en Mato, 2008; 2011). La Organización Indígena de Antioquia (OIA), específicamente desde el Instituto de Educación Indígena en alianza con la Universidad Pontificia Bolivariana y la Universidad de Antioquia, consta de tres ejes de trabajo: 1. La Licenciatura en Etnoeducación, 2. La Escuela de Gobierno y Administración Indígena, y 3. La Licenciatura en Pedagogía de la Madre Tierra (Mato, 2008). Sin embargo, la participación de las mujeres, a pesar de ser estimulada desde las directivas de la OIA, sigue siendo muy inferior a la de los hombres indígenas (sobre 70 participantes en la licenciatura durante 2001-2007, sólo 15 mujeres egresaron [Mato, 2008]). Igualmente, existe el programa de Etnoeducación de la Universidad del Cauca. Según Axel Rojas, encargado de la reconstrucción de la experiencia, hay una Licenciatura en Etnoeducación adscrita al Departamento de Estudios Interculturales de la Facultad de Ciencias Humanas y Sociales de la Universidad del Cauca. También se han venido desarrollando diplomados sobre estudios afrocolombianos, educación ambiental, gestión cultural, gestión etnoeducativa (Mato, 2008). La cifras presentadas, a diferencia de otras 
Superior de Administración Pública (ESAP). Para Ati, al terminar su carrera universitaria, el divorcio era ya un destino posible, y el no crecer bajo la figura de un padre autoritario, sino de una madre empoderadora y liberadora, le permitió un tránsito vital menos traumático hacia su segundo divorcio. Al igual que su madre, debe asumir sola la crianza de su hija.

Durante nuestras conversaciones pudimos observar que en la Escuela de Administración de la que se graduó, como en la mayoría de las instituciones, había un fuerte racismo institucional. Este tipo de prácticas han sido estudiadas por Allard y Santoro (2006), y denominadas “dysconscious racism”. Es decir, una forma de racismo tácitamente aceptado y dominante frente a normas y privilegios que implican a la clase media blanco-mestiza, bajo la forma de éxitos individuales, desconociendo cómo el discurso dominante atraviesa los currículos, los modelos pedagógicos, excluyendo otras formas de conocimiento.

Sin embargo, la universidad se constituye como una herramienta para Ati, quien decide lanzarse al Concejo de Bogotá durante su proceso universitario. Su experiencia permite refutar el esencialismo sobre el determinismo de los roles de género y el predominio de las labores "pastorales de cuidado de las mujeres" analizado por Lumby y Coleman (2007).

No obstante, a nivel personal, se separa por segunda vez, presentándose así una profunda tensión entre carrera y familia. Pero por razones culturales, el cuidado de los hijos y de la familia no aparece como un obstáculo radical, pues las abuelas y otras mujeres de la familia, como las tías y hermanas, juegan un rol fundamental. Sin embargo, lo anterior depende de la autonomía económica de la mujer arhuaca, la cual le permite o no pagar por el cuidado de su familia.

\section{La "entrada en politica"}

La mayoría de los presidentes del país han pasado por el Concejo o la Alcaldía de alguna de las grandes ciudades como Bogotá o Medellín. La entrada de una joven indígena en este escenario, por un partido recién fundado, implicaba muchas dificultades, pero también una estructura de oportunidad política. Su primer obstáculo fue la juventud. Según el Estatuto Orgánico de Bogotá, para ser Concejal se requería tener 25 años. Ati fue electa con 22 años, por lo que al día siguiente de la elección ya estaba demandada. La Corte constitucional falló a favor de ella, protegiendo su derecho fundamental a la

experiencias, muestran cómo en el Cauca han egresado más mujeres que hombres entre los años 2000-2007 (sobre 115 egresados, 68 son mujeres y 47 hombres [Mato, 2008; 2011]). 
identidad de una cultura indígena, y ser representada por un miembro que culturalmente es considerado apto para el cargo.

Sin embargo, el Consejo de Estado consideró que no. A pesar de no entrar al Concejo, este proceso implica para Ati el inicio de su carrera política. Esta experiencia nos hace rememorar muchas de las experiencias descritas por Lumby y Coleman (2007), sobre la existencia de un racismo estructural en las estructuras de poder como la política y la educación. Para esta ocasión, como para su actual candidatura al Senado, la organización arhuaca le dio su apoyo.

Desde ese momento, Ati se perfila como la representante de mujeres y hombres, defensores de causas ecológicas, ambientales y de mujeres. Pero la articulación con los procesos locales se hace muy compleja. Su discurso, su imagen, su historia se convierten en un ícono para colectivos no indígenas, pero como dice el dicho: "Nadie es profeta en su propia tierra". Ati es elegida tras su segunda campaña, siempre apoyada por su madre, dos veces como Concejala de Bogotá.

La siguiente campaña fue muy difícil pero logramos un escaño en el Concejo de Bogotá, no fue nada fácil, porque en ese mismo año se aprobó la reforma política en Colombia y la reforma política castigó a las minorías políticas, es decir, colocó altos umbrales y para poder obtener una curul en un movimiento independiente como indígena era muy difícil. Entonces, la Alianza social Indígena tomó la decisión de no participar en esas elecciones y así llegué yo en representación de los indígenas al Polo Democrático Independiente que estaba surgiendo en ese momento como un movimiento de izquierda, entonces fui electa. Bogotá tiene en el censo más de siete millones de habitantes, pero realmente llegan anualmente en promedio unos dos millones de personas flotantes, migrantes de todo el país, dentro de los que se encuentran las poblaciones indígenas, y se eligen 45 concejales (Entrevista Ati Quigua, Bogotá, 2013).

Durante su paso por el Concejo de Bogotá, como parte del movimiento "Todos Atierra, la común-unidad por la vida y de la red de Arte para la Tierra", Ati hizo alianzas con artistas y músicos de renombre internacional, con el ánimo de generar espacios de interculturalidad, donde las comunidades indígenas presentes en Bogotá pudieran obtener su reconocimiento. ${ }^{8}$ En

7 Iniciativa de la red de Arte para la Tierra y La común-unidad por la Vida Todos Atierra, con la guía de mamos koguis, arhuacos y autoridades espirituales del continente, quienes se han reunido con 52 artistas de Colombia, España y Francia.

8 Impulsó propuestas como el "Estatuto del agua para Bakatá-Bogotá", la política pública indígena distrital, la reforma al estatuto ambiental, la implementación de los cultivos ancestrales andinos como complemento nutricional y la política de soberanía alimentaria sustentada en la economía campesina e indígenas. 
este proceso, su compañero sentimental, el rockero de Doctor Krápula, fue fundamental para el tejido con estos actores.

En el año 2010 convocó a un referendo por los derechos de la naturaleza, iniciativa que ha hecho eco en muchos sectores de la cultura, movimientos sociales y ambientalistas del país. $S e ́$, semilla de vida y paz, es su proyecto político actual, el cual tiene lugar en un contexto político e internacional muy distinto al de su madre. Su formación universitaria y su trabajo político se da en el marco de la adopción por Colombia de la Declaración de los derechos de los pueblos indígenas de las Naciones Unidas aprobada en 2007.

Estos acontecimientos jurídicos y políticos han sido trabajados por autores como Bellier (2013) y Choque (2015). Igualmente, a nivel nacional e internacional, distintos pronunciamientos jurídicos y políticas públicas abogan por la garantía y defensa de los derechos de las mujeres indígenas. Sin embargo, no debe olvidarse que la relación entre derecho y realidad es paradójica, y la experiencia de Ati sigue mostrando prácticas discriminatorias en diferentes niveles.

\section{El salto al Senado}

Después de la tercera campaña para el Concejo, en la cual no sale elegida, decide aspirar al Congreso de la República por la circunscripción especial indígena, en 2013. Constituye una alianza con el indígena amazónico Jaime Estrada de la Organización de los Pueblos Indígenas de la Amazonía Colombiana (OPIAC). Durante su receso político, Ati había iniciado su Doctorado en Derecho en la Universidad del Rosario. Había obtenido una beca de estudios, y su objetivo era trabajar sobre el reconocimiento en Colombia de los derechos de la naturaleza. Inicia su primer semestre.

Inmediatamente, aparecen dos tipos de reacción que varían según los perfiles de los profesores y estudiantes. Aquellos que ven en esta experiencia un reto para generar transformaciones de una de las estructuras universitarias más antiguas y conservadoras del país. Otros justamente piensan que ese no es el lugar para que una indígena perteneciente al mundo político utilice un espacio que debería ser "aprovechado por un jurista ortodoxo". Además de las fuertes prácticas de discriminación racial, ya experimentadas por otros indígenas, ${ }^{9}$ y que por supuesto se reproducen en su caso, su experiencia mues-

9 Norey Quigua, estudiante y líder arhuaco escogido para hacer parte del gobierno universitario, fue descartado en la fase final por su procedencia indígena. 
tra cómo en los niveles más altos de producción del conocimiento se da la imposibilidad de que los saberes indígenas penetren el conocimiento a partir de los estudios interculturales.

En este caso preciso, el de los estudios del derecho constitucional y la protección de los derechos de la naturaleza, pareciera que sólo el investigador que objetiva, y cuyo capital escolar es legítimo puede producir un conocimiento válido (Bourdieu, 1979; Poupeau, 2007; Mato, 2008).

Para finalizar la reconstrucción de las trayectorias es importante resaltar algunas equivalencias y diferencias entre las experiencias de madre e hija. Desde el punto de vista del acceso, la primera generación representada en la madre debe superar más barreras en términos de exclusión, discriminación y falta de oportunidades. Sin embargo, a nivel emocional, la madre a diferencia de su hija, se casa dos veces con indígenas de diversas regiones del país.

Actualmente, ha iniciado una tercera unión con un líder arhuaco. La hija, tras intentar dos veces constituir una familia con parejas indígenas, opta por construir relaciones amorosas con hombres no indígenas. De igual forma, decide tener hasta el momento una sola hija. El acceso a la educación para la segunda generación es más fácil y cuenta con mejores oportunidades.

Pero persisten las barreras culturales desde el mundo arhuaco para reconocer procesos de liderazgo de mujeres arhuacas con trayectorias atípicas. La amalgama entre capital escolar y étnico es muy importante y necesaria. Este último hace énfasis para el caso de las mujeres arhuacas en la dimensión privada de la cultura y exige padrinazgos por parte de hombres de la familia.

\section{Conclusión}

Las historias de estas lideresas nos han permitido aportar datos y reflexiones para la reconstrucción del liderazgo femenino arhuaco, mostrando el rol de la educación superior en la lucha individual y colectiva de estas mujeres. Inspirados en la educación popular feminista, pensamos que si nosotras no contamos estas historias de resistencia, ¿quién lo hará?

A partir del análisis de estas trayectorias podemos concluir que nos enfrentamos a políticas educativas de homogeneización latente, tanto desde lo indígena como desde las instituciones universitarias. Sin embargo, a pesar de las dificultades, estas mujeres ofrecen prácticas políticas y de vida que han resquebrajado el modelo de liderazgo arhuaco actual. La experiencia de estas mujeres es un ejemplo de acción colectiva innovadora que debe seguir siendo analizada con profundidad. 
Para ello, proponemos la noción de capital étnico como una subespecie del capital militante para entender el liderazgo femenino arhuaco que se consolida tras el egreso de la educación superior y el paso por ciertos procesos culturales. Después de reconstruir las trayectorias de madre e hija, vemos que si bien para un líder arhuaco hombre el capital escolar no desempeña un papel fundamental, para el caso de una mujer sí.

No obstante, para el caso de las mujeres existen variaciones importantes. El conocimiento científico o técnico aprendido en las universidades debe estar complementado por un capital étnico fundado en el conocimiento de la cultura y de los procesos organizativos, y el manejo y cuidado de la familia. Deben además estar casadas para ejercer funciones de responsabilidad comunitaria. Al contrario, el paso de los líderes arhuacos al universo de las mujeres indígenas es inexistente. Por el contrario, es observable cómo estas lideresas arhuacas viven al igual que la mayoría de mujeres en Colombia el "doble trabajo", o en su defecto, cuando se lo pueden permitir, promueven inconscientemente la dominación de sus hijas menores o de otras mujeres arhuacas, que requieren recursos propios para su supervivencia.

Igualmente, el racismo institucional y la exclusión de las mujeres arhuacas del mundo académico y político pervive después de casi 25 años del reconocimiento de los derechos especiales de los pueblos indígenas en Colombia. A pesar de los enormes costos emocionales, estas mujeres han aprovechado las estructuras de oportunidad política que sus trayectorias escolares y políticas les han ofrecido. Como lo muestra Gil (2005) en su trabajo, estas mujeres lideran procesos comunitarios articulados a nivel nacional e internacional.

\section{Bibliografía}

Bellier, Irene (2013), Peuples autochtones dans le monde: les enjeux de la reconnaissance, París: Harmmattan.

Bermúdez, Isabel Cristina (2015), La educación de las mujeres en los paises andinos. El siglo $X I X$, Quito: Universidad Andina Simón Bolívar y Corporación Editora Nacional.

Blackmore, Jill (1999), Troubling Women: Feminism, Leadership and Educational Change, Buckingham: Open University Press.

Bosa, Bastien (2015), "Volver: el retorno de los capuchinos españoles al norte de Colombia a finales del siglo XIX” en HiSTOReLo. Revista de Historia Regional y Local, vol. 7, núm. 14, Medellín: Universidad Nacional de Colombia.

Bosa, Bastien (2012), Itinéraires aborigènes - Histoire des relations raciales dans le Sud-Est australien, París: Karthala.

Bosa, Bastien y Eric Wittersheim (2009), Luttes autochtonnes, trajectoires postcoloniales. Amériques, Pacifique, París: Editions Karthala. 
Convergencia Revista de Ciencias Sociales, núm. 70, 2016, Universidad Autónoma del Estado de México

Bourdieu, Pierre (1979), La distinción. Criterio y bases sociales del gusto, Buenos Aires: Taurus.

Bourdieu, Pierre (1989), La noblesse d'état, París: De Minuit.

Bush, Tony et al. (2005), Black and Minority Ethnic Leaders, Final Report to the National College for School Leadership, Lincoln, International Institute for Education Leadership.

Choque, María Eugenia (2015), "Educación Indígena en el marco de los Derechos colectivos en Bolivia", en Educación superior y pueblos indígenas en América Latina. Contextos y experiencias por Daniel Mato, Buenos Aires: UNTREF.

Drucker, Peter (1997), "Foreword", en F. Hesselbein [ed.], The Leader of the Future: New Visions, Strategies and Practices for the Next Era (The Drucker Foundation), San Francisco: CA, Jossey-Bass/Wiley.

Escobar, Arturo, Sonia Álvarez y Evelina Dagnino (2001), Politica cultural y cultura política. Una nueva mirada sobre los movimientos sociales latinoamericanos, Bogotá: TaurusICANH.

Foucault, Michel (2002), Historia de la sexualidad: La Voluntad del Saber, Buenos Aires: Siglo XXI.

Gil, Ángela María (2005), “Tejiendo la vida universitaria en la capital: Nuevos dilemas de la mujer indígena contemporánea”. Tesis para optar al título de Magíster en Antropología Social, Universidad de los Andes, Facultad de Ciencias Sociales, Departamento de Antropología.

Gilligan, Carol (1982), In a Different Voice: Psychological Theory and Women's Development, Cambridge, MA: Harvard University Press.

González, Raquel (2013), Asi cuentas la historia. Mujeres y memoria emberá, Bogotá: Ediciones Gente Nueva.

Gros, Cristian (2000), Politicas de la etnicidad: identidad, Estado y modernidad, Bogotá: ICANH.

Korol, Claudia (2007), "La educación como práctica de libertad. Nuevas lecturas posibles", en Hacia una pedagogía feminista. Géneros y educación popular, Cuadernos de Educación Popular, Buenos Aires: El Colectivo.

Laurent, Virginie (2005), Comunidades indigenas, espacios politicos y movilizaciones electorales en Colombia 1990-1998. Motivaciones, campos de acción e impactos. Bogotá: IFEA-ICANH.

Longo, Roxana (2007), “De eso no se habla”, en Hacia una pedagogía feminista. Géneros y educación popular, Cuadernos de Educación Popular, Buenos Aires: El Colectivo.

Lorde, Audre (1984), Sister outsider: Essays and speeches, Freedom, CA: The Crossing Press.

Lumby Jacky y Marianne Coleman (2007), Leadership and diversity. Challenging theory and practice in education, London: Sage publications.

Mateos, Laura (2015), "Prácticas profesionales, roles comunitarios y saberes-haceres de los gestores interculturales egresados de la Universidad Veracruzana Intercultural”, en Educación superior y pueblos indigenas en América Latina. Contextos y experiencias por Daniel Mato, Buenos Aires: UNTREF. 
Mato, Daniel (2015), Educación superior y pueblos indigenas en América Latina. Experiencias y contextos, Buenos Aires, UNTREF.

Mato, Daniel [coordinador] (2008), Diversidad Cultural e Interculturalidad en Educación Superior. Experiencias en América Latina, Caracas: ASCUN.

Poupeau, Franck (2007), Dominación y movilizaciones. Estudios sociológicos sobre el capital militante y el capital escolar, Córdoba: Ferreyra.

Quijano, Anibal (2000), “Colonialidad del poder, eurocentrismo y América Latina”, en Edgardo Lander [comp.], La colonialidad del saber: eurocentrismo y ciencias sociales. Perspectivas latinoamericanas, Buenos Aires: CLACSO.

Santamaria, Angela (2007), Redes transnacionales y diplomacia indigena. Un estudio transnacional a partir del caso colombiano, Bogotá: Universidad del Rosario.

Ulloa, Astrid et al. [eds.] (2005), Las mujeres indigenas en los escenarios de la biodiversidad, Bogotá: Fundación Natura, Unión Mundial para la Naturaleza (UICN), Grupo de Cultura y Ambiente, y Grupo Cultura y Género (ICAHN).

Wade, Peter (1997), Race and ethnicity in Latin America, Londres: Pluto Press.

\section{Recursos electrónicos}

Allard, Andrea y Ninetta Santoro (2006), “Troubling identities: teacher education students' constructions of class and ethnicity", en Cambridge Journal of Education, vol. 36, núm. 1. Disponible en: http://www.jstor.org/page/journal/compeducrevi/bibliog05.html [1 de mayo 2014].

Chisholm, Linda (2001), "Gender and leadership in South African educational administration”, en Gender and Education, vol. 13, núm. 4. Disponible en: http://www. tandfonline.com/doi/abs/10.1080/09540250120081742?journalCode=cgee20\#previ ew [1 de mayo 2014].

Ken, Ivy (2008), "Beyond the intersection: A new culinary metaphor for race class gender studies”, en Sociological Theory, vol. 26, núm. 2. Disponible en: http://onlinelibrary. wiley.com/doi/10.1111/j.1467-9558.2008.00323.x/abstract [1 de mayo 2014].

Le Bot, Yvon (2004), "Le renversement historique de la question indienne en Amérique Latine”, en Amérique Latine Histoire et Mémoire, Les Cahiers ALHIM, núm. 10. Disponible en: http://alhim.revues.org/100 [2 de febrero de 2005].

Mato, Daniel (2011), "Universidades indígenas de América Latina: Logros, problemas y Desafíos”, en Revista Andaluza de Antropología, núm. 1. Disponible en: http://www. revistaandaluzadeantropologia.org/index.php?page $=$ dmato $[13$ de septiembre de 2015].

Noddings, Nel (1988), "An ethic of caring and its implications for instructional Arrangements", en American Journal of Education, vol. 96. Disponible en: http:// psycnet.apa.org/psycinfo/1989-38107-001 [1 de mayo 2014].

Pratt-Clarke, Menah (2010), Critical Race, Feminism, and Education. A Social Justice Model, New York: Palgrave Macmillan. 
Rabouin, E. Michelle (2000), "Gifting children of promise: Re-imagining the academic margins as transformative legal space”, en Journal Gender Race and Justice, núm. 2. Disponible en: http://ema.sagepub.com/content/31/2/145.short [1 de mayo 2014].

Santamaria, Angela (2008), Redes transnacionales y diplomacia indigena. Un estudio transnacional a partir del caso colombiano, Bogotá: Universidad del Rosario.

Wing, Adrien y Christine Willis (1999), "From Theory to Praxis: Black women, gangs, and critical race feminism", en La Raza Law Journal, vol. 11, núm. 1. Disponible en: http://heinonline.org/HOL/Page ?handle=hein.journals/afamlpol4\&div=3\&g sent $=1 \&$ collection $=$ journals $\# 3$ [ mayo 2014].

\section{Entrevistas}

A Luz Elena Izquierdo, indígena arhuaca de la Sierra Nevada de Santa Marta, asesora del Despacho del Ministro del Interior de Colombia, Bogotá 2012, duración: dos horas.

A Ati Quigua, indígena arhuaca de la Sierra Nevada de Santa Marta, ex concejala de Bogotá, Bogotá abril de 2010, duración: 1 hora y 40 minutos.

A Ati Quigua, indígena Arhuaca de la Sierra Nevada de Santa Marta, ex concejala de Bogotá, Bogotá 2012, duración: 1 hora y 30 minutos.

A Norey Quigua y Dunen Muelas, estudiantes arhuacos de la Universidad del Rosario, hijos de Luz Elena Izquierdo, Bogotá, duración: 1 hora y media.

Angela Santamaria. Doctora en Sociología, Escuela de Altos Estudios en Ciencias Sociales de París (EHESS). Profesora e investigadora en la Universidad del Rosario. Líneas de investigación: acción colectiva, pueblos indígenas y educación superior intercultural. Publicaciones recientes: "Retrospectiva sobre la campaña de solidaridad con las víctimas de Bahía Portete: Un estudio etnográfico del activismo transnacional (2006-2007)”, en Análisis Político, núm. 74, Bogotá: Universidad Nacional de Colombia (2012); "Retrospectiva del proceso constituyente: Un estudio de caso desde la trayectoria de Lorenzo Muelas", en Revista Colombia Internacional, Bogotá: Universidad de los Andes (2013); como coautora en: Acosta Mónica, Naranjo Edgar, Rodríguez Catalina, Rojas Pedro, Santamaria Angela, Identidades porosas, Bogotá: Universidad del Rosario, (2012); Rodríguez Catalina, Rojas Pedro, Santamaria Angela, Memoria, Derecho y Participación: Escuela Intercultural de Diplomacia Indigena: Nabusimake, Sierra Nevada de Santa Martha, Bogotá: Universidad del Rosario (2012).

Recepción: 21 de octubre de 2014.

Aprobación: 26 de agosto de 2015. 